\title{
Postradiation Osteoblastic Osteosarcoma in the Iliac Region: Report of a Case with Multiple Cancers
}

\author{
Marie Aimée Gloria Munezero Butorano ${ }^{1 *}$, Gennaro Baldino ${ }^{1}$, Giulia Orlandini ${ }^{2}$, Clelia Miracco $^{1}$ and Luigi Pirtoli $^{2}$ \\ ${ }^{1}$ Department of Medicine, Surgery and Neuroscience, Section of Pathological Anatomy, Policlinico Santa Maria alle Scotte, Strada delle Scotte 6, 53100 Siena, Italy \\ ${ }^{2}$ Department of Medicine, Surgery and Neuroscience, Section of Radiotherapy, Policlinico Santa Maria alle Scotte, Strada delle Scotte 14, 53100 Siena, Italy
}

*Corresponding author: Marie Aimée Gloria Munezero, Sezione di Anatomia Patologica, Policlinico Santa Maria alle Scotte 6, 53100 Siena, Italy, Tel: 393331543245; E-mail: gbutorano2@hotmail.com

Rec date: Dec 08, 2014, Acc date: Dec 24, 2014, Pub date: Dec 26, 2014

Copyright: (c) 2014 Butorano MAGM, et al. This is an open-access article distributed under the terms of the Creative Commons Attribution License, which permits unrestricted use, distribution, and reproduction in any medium, provided the original author and source are credited.

\begin{abstract}
Although rare, osteosarcoma is the most frequent post-radiation second malignancy of the bone. We present a case of an osteoblastic osteosarcoma that occurred in a woman with a history of two neoplasms, as an example of multiple treatment-associated neoplasms. In fact, prior to the development of this osteosarcoma, our patient had been diagnosed with a breast carcinoma 24 years earlier, which had been treated by quadrantectomy, followed by radiotherapy, but it had recurred after 7 years. Thus, the patient had undergone mastectomy, followed by 4 years of tamoxifen. Then, 14 years later, she developed the second tumor, an endometrial adenocarcinoma, which was treated by surgery and adjuvant radiotherapy. Afterwards, a neoplasm developed in the pelvic bones, which histologically proved to be an osteosarcoma. Athough considered low, there is some risk of developing an endometrial cancer after tamoxifen protracted therapy. Since germline mutation analyses for p53 and RB1 resulted negative in our case, Thus, the treatment could have played a prominent role in our patient.
\end{abstract}

Keywords: Osteoblastic osteosarcoma; Therapy-related cancer; Postradiation sarcoma

\section{Introduction}

Radiation-induced sarcomas of bone are a rare but well recognized, late complication of radiation therapy, accounting for approximately $1.5 \%$ of all bone sarcomas [1]. The latent period between radiation treatment is reported to range from four to fifty-five years, with a mean of eleven years [1].

Treatment with tamoxifen has been associated with an increased risk of endometrial cancer [2], in accord with the selective uptake of tamoxifen by endometrial tissue [2], with its agonistic effects on the endometrium, and with laboratory results [2]. However, the magnitude of the increased risk varies substantially between studies [2].

Our aim is to present a case of an aggressive osteosarcoma that occurred in a 79-year-old woman, 14 years after radiotherapy for an endometrial adenocarcinoma, which, in turn, had developed after 4 years of tamoxifen treatment for a recurrent breast cancer. We suppose that the therapy had a relevant oncogenic role, especially since molecular examinations ruled out RB and p53 germ line mutations in our patient.

\section{Case Report}

A 79-year-old woman came to our attention with a clinical history of pain in the right pelvis, hip, and lower extremity as well as edema of the right lower extremity and small erythematous, round lesions on the skin of her right leg. Twenty-four years earlier, she had undergone a right quadrantectomy for breast cancer, followed by adjuvant radiotherapy. Seven years later, control scans identified a recurrence in the right breast; hence, mastectomy with lymphadenectomy was carried out, followed by tamoxifen treatment for four years. Afterwards she had presented vaginal bleeding. In-depth analyses led to the diagnosis of endometrial adenocarcinoma. Thus, once again she was treated surgically, with hysterectomy, followed by adjuvant radiotherapy with the "box" technique. A total dose of 5040 cGy were administered, 1.8 Gy daily, 5 days a week.

In 2013, 14 years after the last operation and pelvic radiation therapy, a total body CT scan performed after the complaint of severe pelvic pain revealed a large a large area of increased bone density with radiological features of malignancy, involving the right ilioischiopubic ramus and extending to the para-osteal soft tissues (Figure 1A). Histological examination of a fine-needle biopsy from the bone lesion (Figure 1B-E) and of a skin biopsy from one of the cutaneous lesion yielded the same findings: a non-epithelial malignant population of prevalently epithelioid cells, arranged in trabecular and microtrabecular lacy-like structures and with production of an osteoid matrix (Figure 2). Some areas of fused and "small cell"-like cells were also observed. To better characterize the nature of the neoplastic cells, immunohistochemistry was performed using a panel of antibodies (source: podoplanin from DAKO, Milan, Italy; all the other antibodies from Novocastra, Milan, Italy). The neoplastic cells were diffusely and strongly immunopositive for vimentin (SRL33, ready to use), EMA (GP1.4, ready to use), CD 56 (CD564, ready to use), and p53 (DO-7, ready to use). Focal, cytoplasmic and membranous positivities were seen for CD99 (12E7, ready to use). The other tested markers (CK pool [AE1/AE3, 1:100]; CK 7 [RN7, ready to use]; CD 34 [QBEnd/10, ready to use]; podoplanin [Milan, Italy; D2-40, 1:20]; WT1 [WT49, ready to use]; ER [6F11, ready to use]; PgR [clone 16, ready to use]; synaptophysin [27g12, ready to use]; chromogranin A [A5H7, ready to use]) were negative. The final diagnosis was osteoblastic osteosarcoma. The patient was treated with chemotherapy and palliative radiotherapy in D1-D3, nonetheless the lesions extended to the regional lymph 
Citation: Butorano MAGM, Baldino G, Orlandini G, Miracco C, Pirtoli L (2014) Postradiation Osteoblastic Osteosarcoma in the lliac Region: Report of a Case with Multiple Cancers. J Cytol Histol 6: 297. doi:10.4172/2157-7099.1000297

Page 2 of 3

nodes and metastasized to the lungs and the patient died nine months

later.

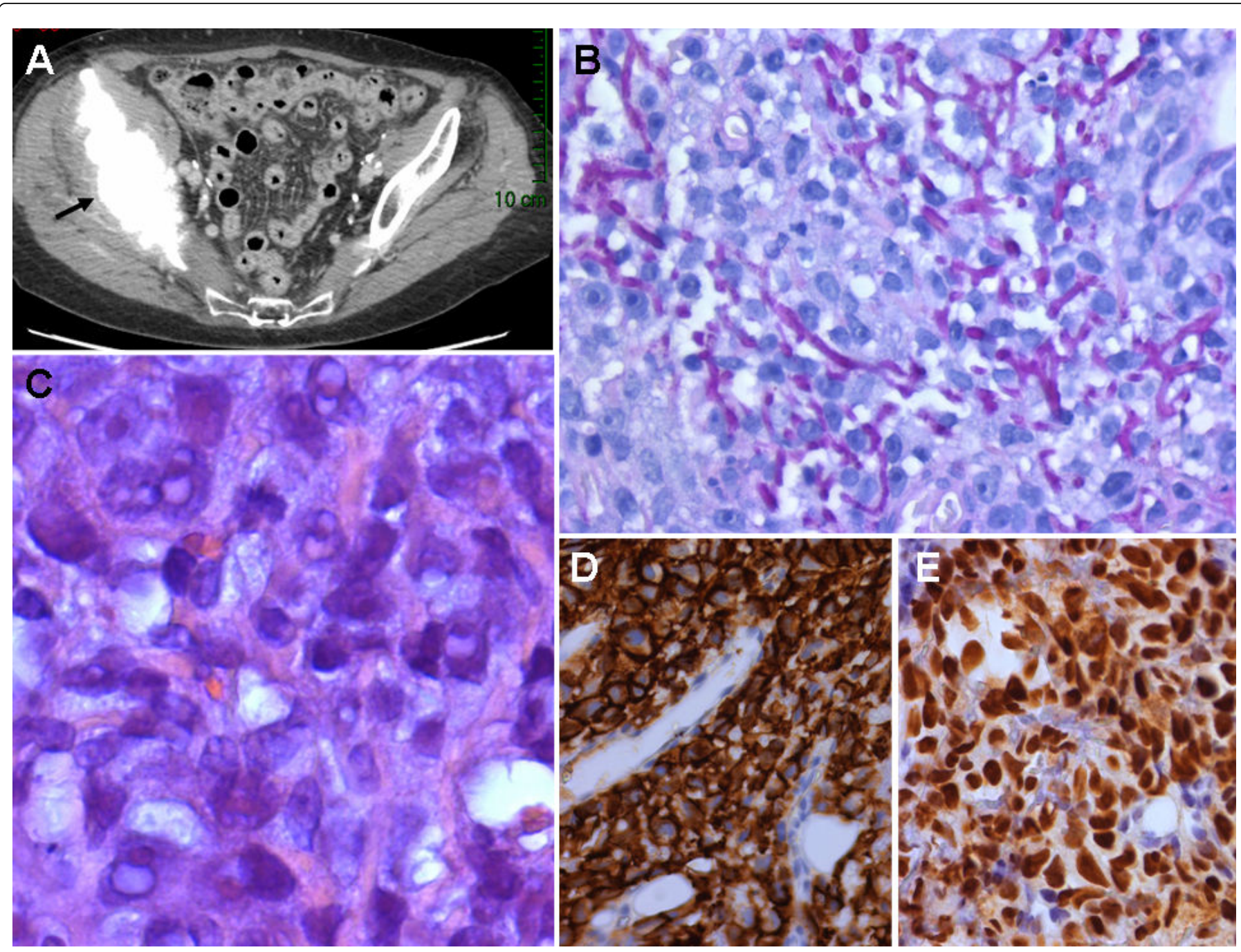

Figure 1: Features of the lesion: a vast, osteocondensant lesion of the pelvic bones associated with osteo-calcific degeneration of the peri- and paraosteal tissues (A, arrow). PAS diastase stain (B) underlines a lacelike osteoid bone matrix, produced by a highly cellular neoplasm. Detail of atypical tumor cells (C), strongly and diffusely positive for CD56 (D). Virtually all tumor cell nuclei are positive for p53 (E). B-E: Original Magnification x 400; B: PAS after diastase digestion; C-E: immunohistochemistry with diaminobenzidine chromogen.

\section{Discussion}

Our patient developed an osteoblastic osteosarcoma 14 years after irradiation of the pelvis for a uterine adenocarcinoma that had appeared after tamoxifen treatment for recurrence of a breast cancer. Although our case does not differ much from those already reported in literature, we want to highlight the risk of cancerogenesis that is associated with adjuvant therapies, carried out in our case for two distinct neoplasms. Moreover, we want to point that although it is still controversial whether tamoxifen does increment the risk of developing uterine neoplasms, in our case the patient developed endometrial adenocarcinoma after tamoxifen treatment.

Post-radiation sarcomas are rare complications of radiotherapy that occur within a previously irradiated field after several years of latency [3]. Post-radiation sarcoma accounts for $0.5 \%$ to $5.5 \%$ of all sarcomas
[3 of Noh] and $50-60 \%$ of radiation-induced sarcomas are osteosarcomas [4]. It is estimated that the risk of developing osteosarcoma in irradiated bone is $0.03-0.08 \%$ [4]. It can develop in any irradiated bone, with most common locations being the pelvis and the shoulder [4].

Mutations of the p53 gene, involved in the maintenance of genomic integrity, have been found in most cases of radiation-induced sarcomas [5,6]. In addition to p53, the retinoblastoma gene (RB1) has also been implicated in the tumorigenesis of both sporadic and radiation-induced osteosarcoma [6,7]. Secondary sarcomas occur more commonly in patients with hereditary retinoblastoma, suggesting a genetic role in their etiology [5]. In our case, molecular analyses were carried out, but no germline mutation in the p53 nor in the RB1 gene were found, thus radiotherapy would have played a prominent role. 
Citation: Butorano MAGM, Baldino G, Orlandini G, Miracco C, Pirtoli L (2014) Postradiation Osteoblastic Osteosarcoma in the lliac Region: Report of a Case with Multiple Cancers. J Cytol Histol 6: 297. doi:10.4172/2157-7099.1000297

Page 3 of 3

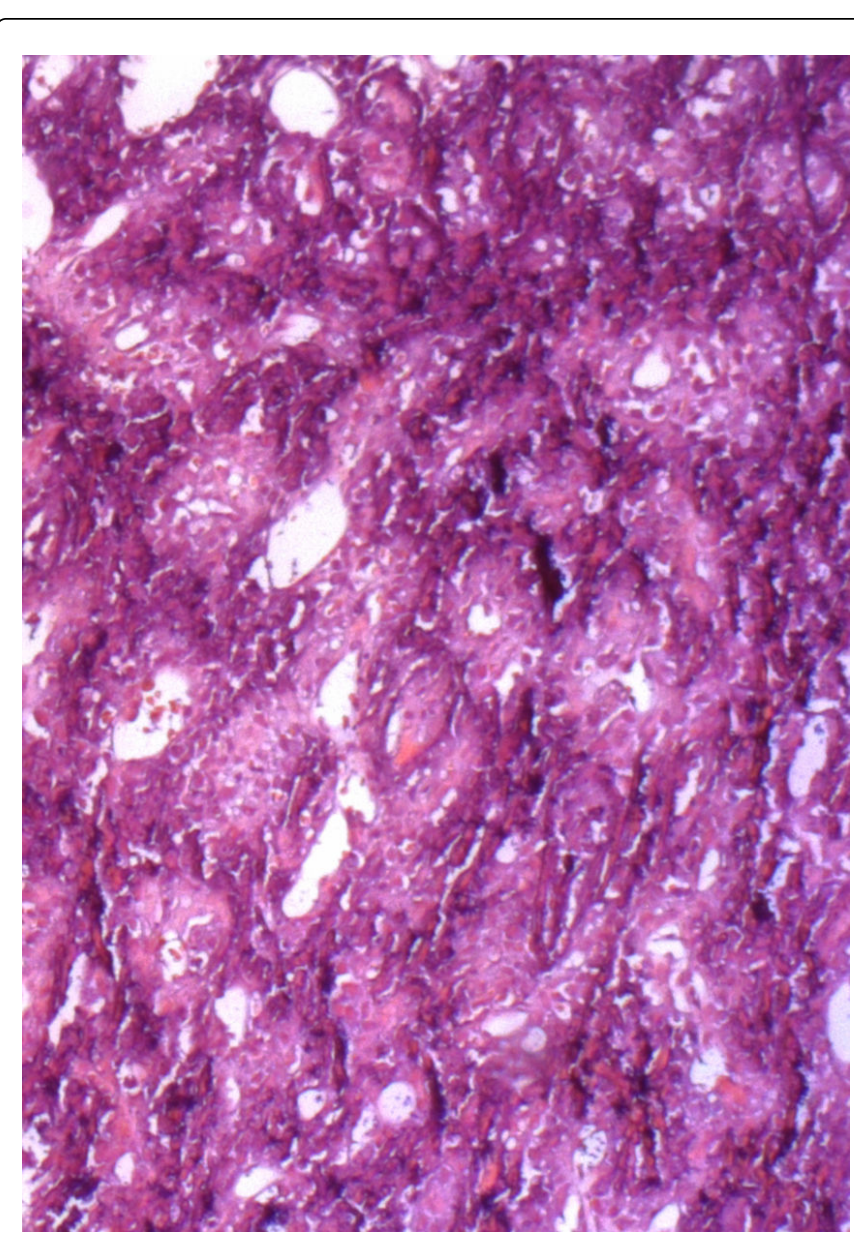

Figure 2: Osteoblastic osteosarcoma: an image at low magnification of this bone-forming tumor.

Original Magnification x 50; Hematoxylin and Eosin.

Exposure to ionizing radiation, in fact, causes directly damages DNA by the generation of free radicals [5], and therapeutic radiation is the most common cause of radiation-induced bone sarcomas $[5,8]$. Whereas sub-lethal doses of radiation from an external source generally do not induce bone tumors, high-dose megavolt radiotherapy is known to be a risk factor for secondary bone tumor development [8].

There are no distinguishable imaging features of post-radiation sarcomas [3]. However, the presence of bony destruction with a soft tissue mass, tumor matrix mineralization at a previously irradiated area, and an appropriate latency period could be important clues for a diagnosis of post-radiation sarcoma [3].

Osteosarcoma arises from primitive mesenchymal bone-forming cells. Histologically, its hallmark is the production of osteoid, an unmineralized bone matrix that histologically appears as eosinophilic, dense, homogenous, amorphous and curvilinear intercellular material, somewhat refractile [4]. In osteoblastic osteosarcoma, the bone/ osteoid matrix contains pleomorphic malignant cells and coarse neoplastic woven bone [4]. The neoplastic bone varies in quantity and is deposited as primitive, disorganized trabecular and coarse, lace-like patterns, thin, arborizing lines of osteoid interweaving between neoplastic cell, as we observed in our case, or broad, large sheets of coalescing, trabeculae [4]. We also observed a strong positivity for CD56, a neuroectodermal marker usually expressed by aggressive osteosarcomas [9].

Radiation-induced sarcoma is, in fact, much more aggressive than its naturally occurring counterpart [5], with 5-year survival rates of $27 \%$ to $36 \%$ [3]. Only complete surgical resection can guarantee longterm survival [8]. As is the case for other types of therapy-associated secondary tumours, most studies reported a worse response to chemotherapy and a less favorable outcome compared to primary bone sarcomas having comparable morphology [8]. Adverse prognostic factors include a patient over 40 years of age, metastases at presentation, as it was in our case, and central location of the tumour [5]. Moreover, a low incidence after a long latency period makes it difficult to diagnose the disease accurately [3]. In our case in fact, the patient had been quite well and when the osteosarcoma was discovered, it was so advanced that neither surgery, chemo- nor or radiotherapy could yield relief. Thus, we feel that reports of therapyinduced secondary neoplasms could increase clinicians' concern and awareness on adjuvant treatment risks, for an accurate and early diagnosis.

\section{References}

1. Daliwal J, Sumathib VP, Grimera RJ (2010) Radiation-induced periosteal osteosarcoma. Grand Rounds 10: 13-18.

2. Swerdlow AJ, Jones ME; British Tamoxifen Second Cancer Study Group (2005) Tamoxifen treatment for breast cancer and risk of endometrial cancer: a case-control study. J Natl Cancer Inst 97: 375-384.

3. Noh JM, Huh SJ, Choi DH, Park W, Nam SJ (2012) Two cases of postradiation sarcoma after breast cancer treatment. J Breast Cancer 15: 364-370.

4. Trihia H, Valavanis C (2012) Histopathology and Molecular Pathology of Bone and Extraskeletal Osteosarcomas. In: Agarwal A (edn) Osteosarcoma.

5. Kalra S, Grimer RJ, Spooner D, Carter SR, Tillman RM, et al. (2007) Radiation-induced sarcomas of bone: factors that affect outcome. J Bone Joint Surg Br 89: 808-813.

6. Broadhead ML, Clark JC, Myers DE, Dass CR, Choong PF (2011) The molecular pathogenesis of osteosarcoma: a review. Sarcoma 2011: 959248.

7. Gonzales-Vasconcellos I, Anastasov N, Sanli-Bonazzi B, Klymenko O, Atkinson MJ, et al. (2013) Rb1 haploinsuffiency promotes telomere attrition and radiation-induced genomic instability. Cancer Res 73: 4247-4255.

8. Rümenapp C, Smida J, Gonzalez-Vasconcellos I, Baumhoer D, Malfoy B, et al. (2012) Secondary radiation-induced bone tumours demonstrate a high degree of genomic instability predictive of a poor prognosis. Curr Genomics 13: 433-437.

9. Folpe AL, Gown AM (2008) Immunohistochemistry for analysis of soft tissue tumors. pp. 129-174. In: Weiss AW, Goldbluma JR (eds), Enzinger \& Weiss's Soft Tissue Tumors. Mosby Elsevier, St Louis, USA. 